\title{
Synthetic Lethality Triggered by Combining Olaparib with BRCA2- Rad51 Disruptors
}

\author{
Federico Falchi, ${ }^{\dagger}$ Elisa Giacomini, ${ }^{\dagger}$ Tiziana Masini, ${ }^{\dagger}$ Nicolas Boutard, ${ }^{\dagger}$ Lorenza Di Ianni, ${ }^{\ddagger}$
} Marcella Manerba, ${ }^{\ddagger}$ Fulvia Farabegoli, ${ }^{\S}$ Lara Rossini, ${ }^{\prime \prime}$ Janet Robertson, " Saverio Minucci, ${ }^{\perp, \#}$ Isabella Pallavicini, ${ }^{\perp}$ Giuseppina Di Stefano, ${ }^{\ddagger}$ Marinella Roberti, ${ }^{\S}$ Roberto Pellicciari, ${ }^{\|}$ and Andrea Cavalli*, $\dagger, \S(1)$

${ }^{\dagger}$ CompuNet, Istituto Italiano di Tecnologia, via Morego 30, I-16163 Genova, Italy

${ }^{\ddagger}$ Department of Experimental, Diagnostic and Specialty Medicine, University of Bologna, Via S. Giacomo 14, I-40126 Bologna, Italy

${ }^{\S}$ Department of Pharmacy and Biotechnology, University of Bologna, Via Belmeloro 6, I-40126 Bologna, Italy

${ }^{\|}$TES Pharma S.r.l., Via Palmiro Togliatti 22bis, I-06073 Loc. Terrioli, Corciano, Perugia, Italy

${ }^{\perp}$ Department of Experimental Oncology at the European Institute of Oncology, IFOM-IEO Campus, Via Adamello 16, I-20100 Milan, Italy

${ }^{\#}$ Department of Biosciences, University of Milan, Via Celoria 26, I-20100 Milan, Italy

\section{Supporting Information}

ABSTRACT: In BRCA2-defective cells, poly(adenosine diphosphate $[\mathrm{ADP}]$-ribose) polymerase inhibitors can trigger synthetic lethality, as two independent DNA-repairing mechanisms are simultaneously impaired. Here, we have pharmacologically induced synthetic lethality, which was triggered by combining two different small organic molecules. When administered with a BRCA2-Rad51 disruptor in nonmutant cells, Olaparib showed anticancer activity comparable to that shown when administered alone in BRCA2-defective cells. This strategy could represent an innovative approach to anticancer drug discovery and could be extended to other synthetic lethality pathways.
Small-molecule-induced synthetic lethality

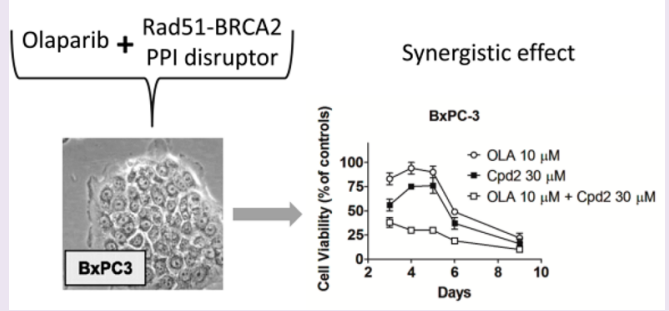

Synth ynthetic lethality is the lethal phenotype arising from a combination of two gene mutations, which do not affect cell viability when they occur individually. ${ }^{1,2}$ In principle, targeting the synthetic lethal partner of an altered gene in cancer should selectively kill cancer cells while sparing normal cells. One straightforward application of synthetic lethality in anticancer drug development is the use of poly(adenosine diphosphate $[\mathrm{ADP}]$-ribose) polymerase (PARP) inhibitors in BRCA2-defective oncology patients. BRCA2 mutations are undesirable because they are associated with tumorigenesis (the so-called "BRCAness" tumors). ${ }^{3}$ However, they can impair DNA-repairing mechanisms and improve cancer cells' sensitivity to DNA-damaging chemotherapeutics and PARP inhibitors. ${ }^{4}$ Olaparib (Lynparza, developed by AstraZeneca) was the first PARP inhibitor approved in 2014 to treat women with advanced ovarian cancer associated with defective BRCA genes. $^{5}$ Several clinical trials are studying Olaparib for breast and pancreatic BRCAness tumors, and these strategies rely on the synthetic lethality paradigm. ${ }^{6,7}$ Inhibiting PARP enzymes allows the accumulation of single-strand breaks, which are subsequently converted into DNA double-strand breaks (DSBs). Normal cells can efficiently repair DSBs. But when homologous recombination (HR) is impaired, as with BRCAness tumors, DSBs cannot be mended, leading to the selective killing of cancer cells. ${ }^{8}$ Of the core proteins involved in HR, Rad51 is an evolutionarily conserved recombinase enzyme that plays a key role in recombination. It multimerizes on the $3^{\prime}$ tailed single-strand DNA overhangs, forming nucleofilaments that are responsible for seeking homologous DNA sequences and subsequent strand-exchange invasion. ${ }^{9}$ Rad51 is recruited to the DSB site by BRCA2 through a protein-protein interaction mediated by eight well-conserved motifs, known as BRC repeats. ${ }^{10-12}$ In BRCA2-defective cells, impaired recruitment of Rad51 means that $H R$ is not properly performed, and so DSBs are not efficiently repaired.

Here, we report on a new anticancer drug discovery concept, which combines BRCA2-Rad51 disruptors with Olaparib in order to trigger synthetic lethality exclusively using small organic molecules. To identify disruptors of this proteinprotein interaction, we performed a virtual screening protocol based on high-throughput docking using the X-ray crystal structure of BRC4 (the fourth BRC repeat) in complex with the catalytic domain of Rad51 (PDB code: 1NOW). ${ }^{13}$ BRC4 binds Rad51 in two different hydrophobic pockets (Figure 1a). ${ }^{14,15}$

Received: August 15, 2017

Accepted: August 25, 2017

Published: August 25, 2017 


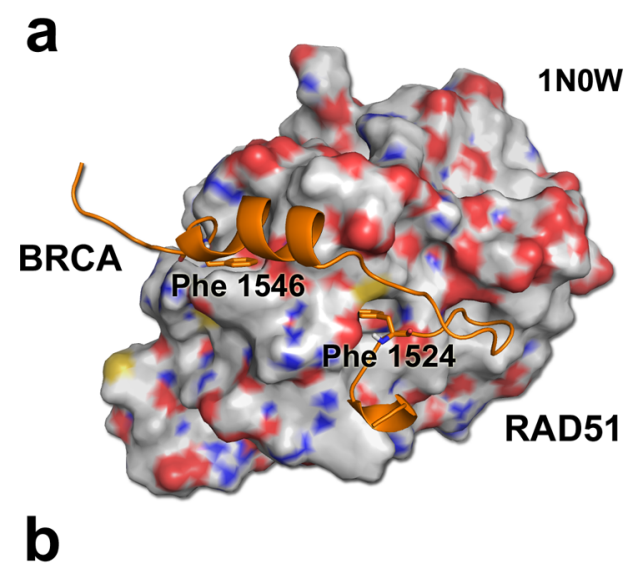

C
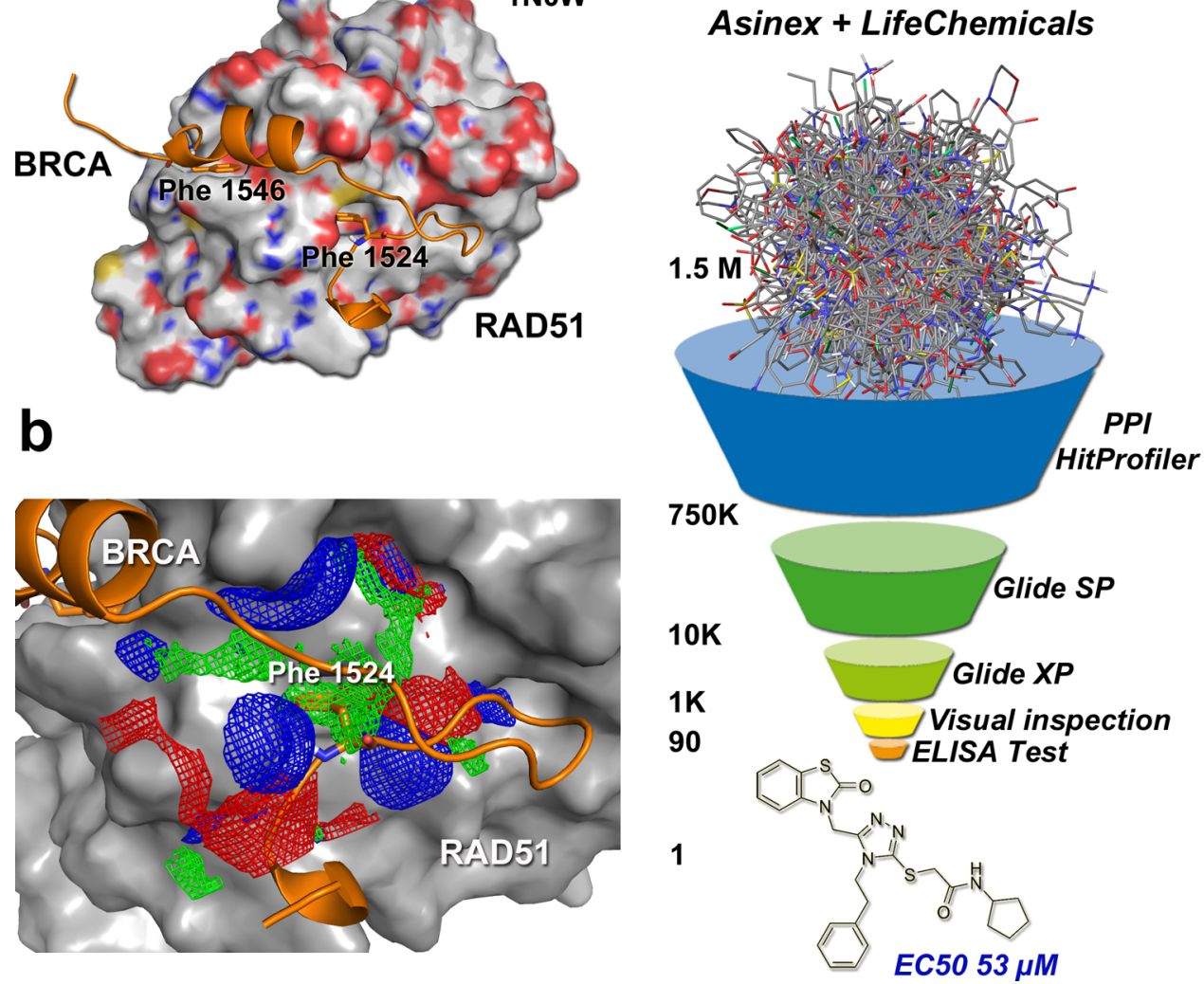

Figure 1. (a) Rad51-BRCA2 BRC repeat complex. Rad51 is represented as a surface, BRCA as a cartoon. The two hot spots of the interaction between the proteins (Phe1524 and Phe1546) are highlighted in sticks. (b) SiteMap hydrophobic (green), hydrogen-bond donor (blue), and hydrogen-bond acceptor (red) maps for 1N0W in the proximity of Phe1524. (c) Schematic representation of the virtual screening protocol. The numbers on the left column represent the number of compounds in each step; the filters used to narrow down the compounds are reported on the right column.

One pocket can lodge BRC4's "FxxA" motif and is critical for Rad51 multimerization (Phe1524 of BRC4 or Phe86 of Rad51 can both bind into this pocket). The other pocket can lodge the "LFDE" motif far from the oligomerization interface. Highthroughput docking was carried out using the "FxxA" pocket (Figure 1b), according to the protocol reported in Figure 1c. Ninety small molecules were selected, purchased, and tested for their inhibitory activity using a competitive biochemical ELISA assay, as previously described by Rajendra et al. ${ }^{14}$ This assay is an efficient tool for directly measuring inhibition of the BRC4Rad51 interaction, at a molecular level. Indeed, when one of our compounds physically interacts with Rad51, BRC4 is displaced as a consequence of the disruption of the BRC4Rad51 molecular interaction.

Of the tested compounds, 1 (Figure 1c and Table 1) was the best candidate in terms of $\mathrm{EC}_{50}$ and chemical tractability, and its activity was confirmed by retesting the newly synthesized compound 1. Preliminary structure-activity relationship (SAR) studies focused on the ethyl phenyl region to find the proper length of the alkyl chain between the triazole and the phenyl ring. The synthesis of compounds $\mathbf{1 - 4}$ is reported in the Supporting Information. A large library of analogues along with extensive SAR studies will be described in a subsequent medicinal chemistry paper. Compound 2 (Table 1), with a propyl phenyl fragment, showed slightly better biological activity than the initial hit compound and was selected for further biological studies.
To test the hypothesis of pharmacologically induced synthetic lethality, we studied whether the combination of Olaparib with 2 could increase the efficacy of PARP inhibitors in cells expressing the functional BRCA2 protein. These results were compared to those obtained using BRCA2-defective cells. Given pancreatic cancer's lethality and the availability of a cell line lacking a functional BRCA2 (the Capan-1 cell line), we selected pancreas adenocarcinoma as a cellular model. The functional BRCA2 cell line was BxPC- 3 because, aside from the BRCA2 mutation, these cells match Capan-1 well in terms of tumor type and differentiation state. ${ }^{16}$ First, we evaluated Olaparib's effects on the viability of the two cultures. The cell lines were exposed to scalar doses of the drug for 1-7 days. The effects observed at day 7 are summarized in Figure 2a. As expected, Olaparib was more active against BRCA2-defective cells (i.e., Capan-1) relative to BxPC-3. We then selected a 10$\mu \mathrm{M}$ concentration to investigate the drug in combination with 2.

Figure $2 \mathrm{~b}$ and $\mathrm{c}$ show the effects caused by 2 on the viability of BxPC-3 and Capan-1 cells, respectively. The compound was tested at scalar doses $(0-50 \mu \mathrm{M})$ for a period of 7 days. Notably, the culture expressing the functional BRCA2 appeared to be more responsive to the compound.

Then, we focused on the combination of Olaparib with 2 . Figure 2d,e report the results. In Capan-1 cells, the combination with $\mathbf{2}$ did not affect Olaparib toxicity. Conversely, in BxPC-3 cells, administering 2 at concentrations of $20 \mu \mathrm{M}$ 
Table 1. $\mathrm{EC}_{50}$ of 1, 2, 3, and 4 on ELISA Assay ${ }^{a}$

\begin{tabular}{|c|c|c|}
\hline Compound & Structure & $\mathrm{EC}_{50}{ }^{*}$ \\
\hline 1 & & $\mathrm{EC}_{50}=53 \pm 3 \mu \mathrm{M}$ \\
\hline 2 & & $\mathrm{EC}_{50}=25 \pm 2 \mu \mathrm{M}$ \\
\hline 3 & & N.A. ${ }^{* *}$ \\
\hline 4 & & N.A.** \\
\hline
\end{tabular}

${ }^{a}(*)$ All points were tested in triplicate with error bars indicating the standard deviation. $(* *)$ Not active.

and $30 \mu \mathrm{M}$ significantly increased the drug's effects. In these samples, evaluation of the combination index suggested a potentially synergic effect rather than a simple additive effect between the two molecules (Figure S1). This was likely due to the simultaneous impairment of two DNA repair mechanisms, eventually resulting in a pharmacologically induced synthetic lethality. Similar results were recently obtained by Jdey et al., who investigated oligonucleotides in combination with Olaparib and other PARP inhibitors. ${ }^{17}$

To strengthen the evidence that 2 inhibits the BRCA2Rad51 signaling pathway, cell viability experiments were also performed on BxPC-3 cells with stable Rad51 silencing (Figure S2). Results were evaluated after $96 \mathrm{~h}$ of incubation with the compounds, when the highest effects had been observed on the parental culture (Figure 2d). As reported in Figure 2f, abrogation of Rad51 expression in BxPC-3 cells was found to cancel the potentiating effect produced by $\mathbf{2}$ on Olaparib citotoxicity.

In further studies, we verified whether administering 2 increased the DNA damage signs produced in Capan-1 and BxPC-3 cells by Cisplatin. DNA damage was evaluated by assessing $\mathrm{H} 2 \mathrm{AX}$ phosphorylation $(\gamma \mathrm{H} 2 \mathrm{AX})$. Cell cultures were exposed to $12 \mu \mathrm{M}$ Cisplatin for $1 \mathrm{~h}$. The drug was then removed, and $2(20 \mu \mathrm{M}$ and $30 \mu \mathrm{M})$ was added to the culture medium. We preliminarily verified that $1 \mathrm{~h}$ treatment with 12 $\mu \mathrm{M}$ Cisplatin caused DNA damage signs, which became evident within $24 \mathrm{~h}$ and tended to reduce during the following $24 \mathrm{~h}$. In the cell cultures exposed to Cisplatin plus 2, DNA damage was evaluated at $48 \mathrm{~h}$. Figure $2 \mathrm{~g}$ shows the densitometric reading of phospho-H2AX levels, evidenced by immunoblotting. The vertical lines indicate the samples for comparison. The blotted membrane image can be seen in Figure S4. In Capan-1 cells, 2 did not affect Cisplatin-induced DNA damage. Conversely, an increased $\gamma \mathrm{H} 2 \mathrm{AX}$ signal was observed in the BxPC-3 cell samples treated with the $2 /$ Cisplatin combination. Although not statistically significant, this increase is in line with data obtained in the cytotoxicity experiments for the 2/Olaparib combination. We also sought to demonstrate that 2's effect (concentration of $20-30 \mu \mathrm{M}$ ) on $\gamma \mathrm{H} 2 \mathrm{AX}$ activation could be similar to that of Rad51 gene knockdown. A procedure for transient Rad51 silencing was adopted in this case (Figure S3). Results are shown in Figure $2 \mathrm{~h}$. The presence of $\gamma \mathrm{H} 2 \mathrm{AX}$ indicates that some of the DNA damage remains unrepaired and may contribute to cancer cell death. Constitutive DNA damage without any genotoxic insult can be present to a varying extent in cancer cell lines. ${ }^{18}$ Rad51-defective cells exhibited a marked increase in the frequency of spontaneous and chromatid type breaks, indicating Rad51's vital role in the DSB repair pathway. ${ }^{19}$ Here, $\gamma \mathrm{H} 2 \mathrm{AX}$ focus formation increased significantly after Rad51 gene silencing ( $p<0.05$, Figure $2 \mathrm{i}$ ). Figure $2 \mathrm{i}$ also shows that the effect of Rad51 gene silencing on $\gamma \mathrm{H} 2 \mathrm{AX}$ was comparable to that of treatment with 2 . No statistically significant difference was observed in the number of $\gamma \mathrm{H} 2 \mathrm{AX}$-marked nuclei between the Rad51-silenced and the 2treated cultures. Finally, to assess potential off-target activities of 2 , a few proteases and phosphatases were selected and tested, as related structures have the potential to inhibit these enzyme families. As shown in Table 2, compound 2 was rather clean and totally inactive against a set of selected proteases and phosphatases, which could play a role in the cell-based anticancer activity here investigated.

Clinical evidence has shown that PARP inhibitors are more effective in oncology patients carrying BRCA2 mutations than in nonmutated individuals. ${ }^{20}$ Mutated BRCA2 protein partially loses its ability to bind Rad51, resulting in incompetent repair of damaged DNA in cancer cells. ${ }^{13}$ As such, we hypothesized that the enhanced sensitivity in BRCA2-defective oncology patients could be mimicked pharmacologically in nonmutated individuals by administering disruptors of the BRCA2-Rad51 interaction. This would make cancer cells more sensitive to PARP inhibitors, widening the spectrum of possible applications of these anticancer drugs. We have here demonstrated that combining Olaparib with a small molecule BRCA2-Rad51 disruptor does indeed increase the sensitivity of nonmutated pancreatic cancer cells, making them as responsive to Olaparib as BRCA2-defective cell lines. Furthermore, our analyses have shown a synergistic effect (rather than a simple additive effect) between BRCA2-Rad51 disruptors and Olaparib, which is likely a consequence of the simultaneous inhibition of two DNA-repair mechanisms. Oncology patients carrying BRCA2 mutations also show enhanced sensitivity to some DNAdamaging chemotherapy agents, including platinum derivatives, topoisomerase inhibitors, 5-fluorouracil, and alkylating drugs. ${ }^{21}$ Combining BRCA2-Rad51 disruptors with these drugs could improve efficacy in treating nonmutated patients, broadening the potential applications of these drugs in anticancer chemotherapy. Finally, BRCA2-Rad51 disruptors could potentially be used to treat patients who develop resistance to PARP inhibitors, which is mostly due to either restoration of 

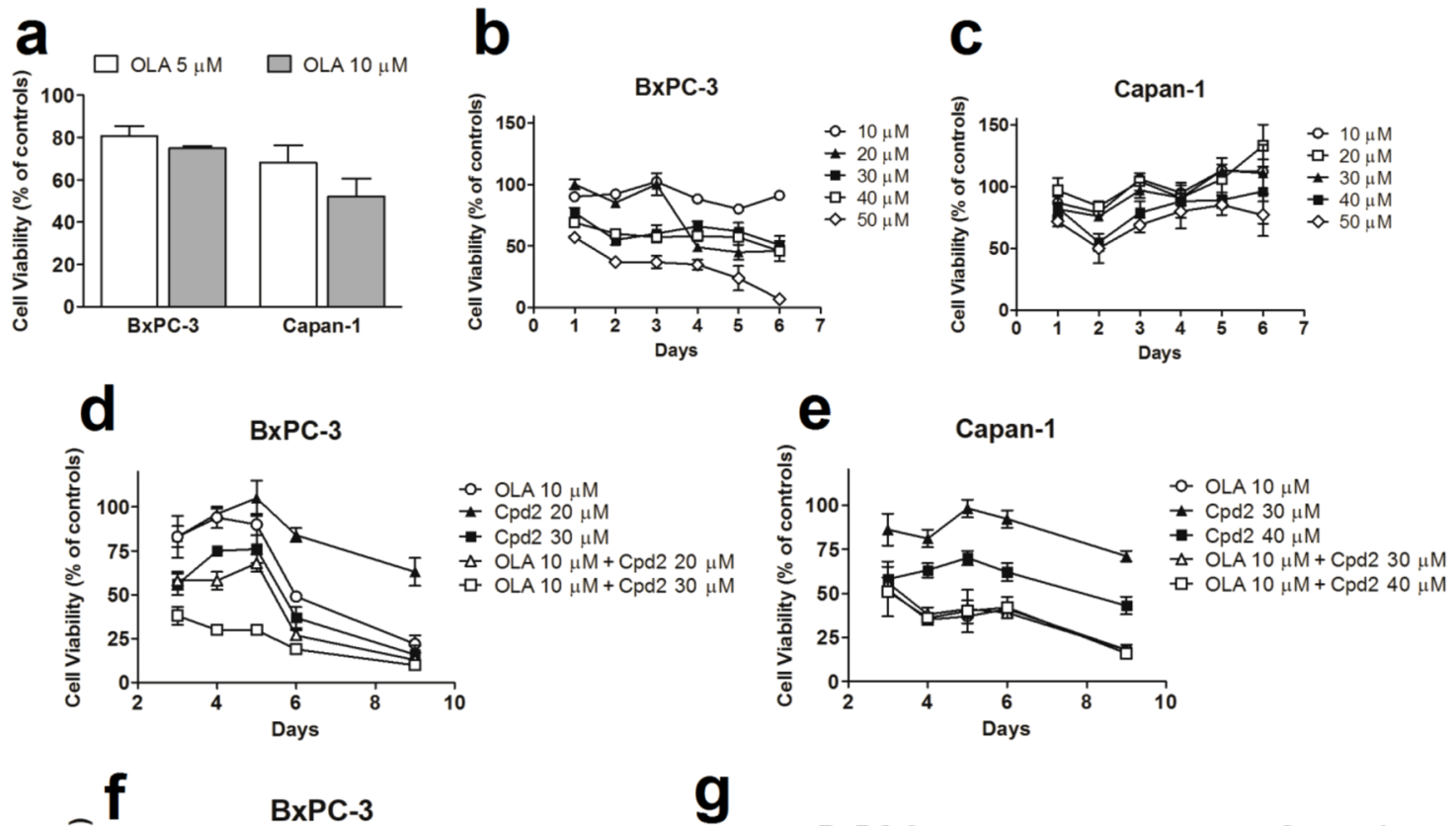
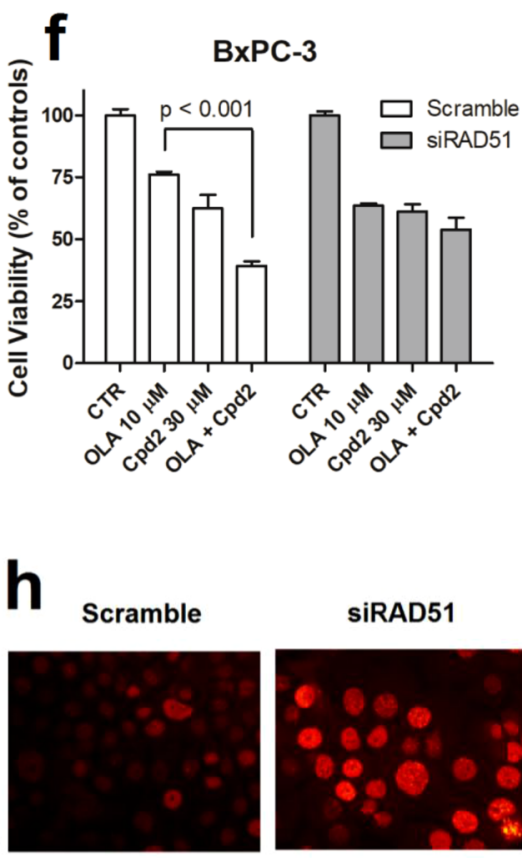

siRAD51

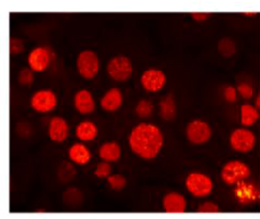

$\gamma \mathrm{H} 2 \mathrm{AX}$
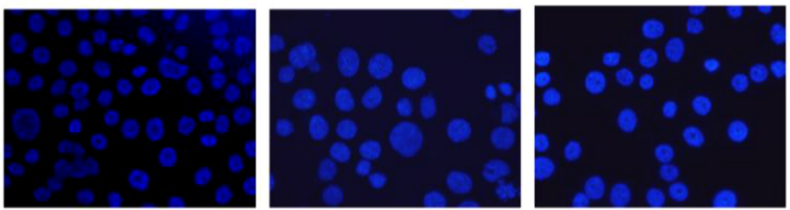

BxPC-3

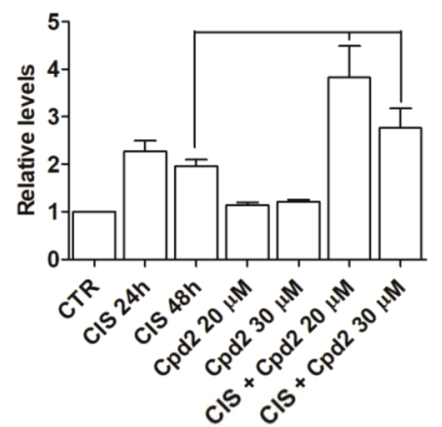

Cpd2 $30 \mu \mathrm{M}$

Capan-1

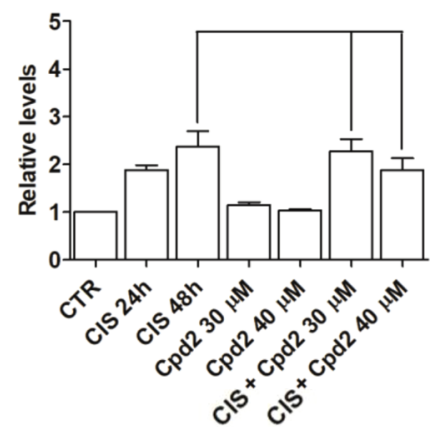

i
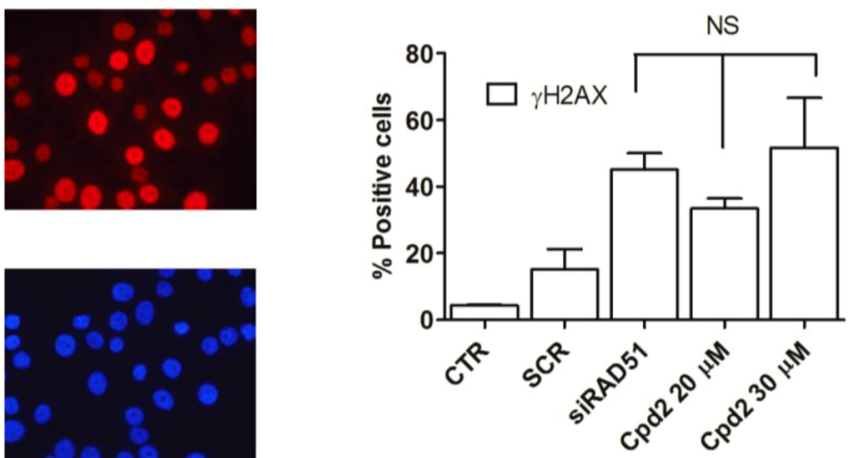

DAPI

Figure 2. Experiments on cell cultures. (a) Effect of 7 days of Olaparib treatment on the viability of BxPC-3 and Capan-1 cells. (b, c) Dose-response curves assessed in BxPC-3 (b) and Capan-1 (c) cells exposed to 2. The compound doses used for combination experiments have been sketched with dark symbols. (d, e) Combination experiments of Olaparib with 2 in BxPC-3 (d) and Capan-1 (e). In the BxPC-3 cell line, the results obtained with Olaparib were compared with those obtained with Olaparib in combination with 2 using two-way ANOVA; for both 20 and $30 \mu \mathrm{M} 2, p$ values $<$ 0.001 were obtained at 3-6 days of treatment. (f) Combination experiment of Olaparib with 2, in BxPC-3 cells after stable Rad51 silencing, compared with scrambled controls. The effect was evaluated after a $96 \mathrm{~h}$ treatment. Results obtained in siRad 51 cultures were statistically analyzed by ANOVA followed by Bonferroni post test. (g) Densitometric reading of immunoblots evaluating $\gamma \mathrm{H} 2 \mathrm{AX}$ expression (normalized on Actin levels) in cells treated with Cisplatin $(12 \mu \mathrm{M})$ and 2 at the same doses used in the Olaparib combination experiments. For a detailed explanation, see the text. Vertical lines indicate the samples for comparison. (h) Immunofluorescence $\gamma \mathrm{H} 2 \mathrm{AX}$ detection in nuclei of BxPC-3 cells treated with RAD51 siRNA or 2. The percentage of positive cells are reported in the graph of frame (i).

nonmutated BRCA2 protein $^{22}$ or overexpression of Rad51, ${ }^{23}$ which in turn increases HR. Indeed, Rad51 is overexpressed in a wide variety of cancers, including breast cancer, and is a mechanism for increasing HR in cancer cells. ${ }^{24}$ Since several 
Table 2. Enzyme Inhibition Activity for Compound 2 in CEREP Assays against Some Potential Off-Targets ${ }^{a}$

\begin{tabular}{|c|c|c|}
\hline enzyme & $\begin{array}{c}\% \text { inhibition at } 25 \\
\mu \mathrm{M}\end{array}$ & references \\
\hline cathepsin B (h) & 1.9 & 26 \\
\hline cathepsin D (h) & -6.6 & 27 \\
\hline cathepsin E & -25.8 & 27 \\
\hline cathepsin S (h) & 12.3 & 28 \\
\hline MMP-2 (h) & -2.9 & 29 \\
\hline phosphatase CDC25A (h) & -5 & 30 \\
\hline phosphatase CDC25B (h) & -0.3 & 30 \\
\hline $\begin{array}{l}\text { protein serine/threonine phosphatase, } \\
\text { PP2A }\end{array}$ & 5.5 & 31 \\
\hline $\begin{array}{l}\text { protein tyrosine phosphatase, PTPRC } \\
\text { (CD45) }\end{array}$ & -2.9 & 32,33 \\
\hline
\end{tabular}

${ }^{a}$ Results showing an inhibition or stimulation higher than $50 \%$ are considered to represent significant effects of the test compound.

anticancer chemotherapy agents cause DSBs, inhibiting HR could impact cancer cell survival in a major way and may increase sensitivity to DNA-damaging agents or radiotherapies.

In conclusion, we have demonstrated that, when administered in combination, Olaparib and a BRCA2-Rad51 disruptor can lead to anticancer activity, which is very similar to that observed in BRCAness tumors treated with PARP inhibitors. This new paradigm is based on the possibility to trigger synthetic lethality by exclusively using small organic molecules. In addition to opening innovative avenues for treating cancer in BRCA2-mutated and nonmutated patient populations, this paradigm could also be extended to other gene pairs involved in diverse synthetic lethality pathways.

\section{METHODS}

ELISA Assay. Competitive ELISA screening assays using biotinylated BRC4 peptide to disrupt the BRC4-RAD51 interaction were performed by modifying the method described by Rajendra and Venkitaraman. ${ }^{14}$

BRC4-biotinylated peptide (N-term BiotinKEPTLLGFHTASGKKVKIAKESLDKVKNLFDEKEQ from Life Technologies) was used to coat 384 well plates (Nunc). After washing with PBS containing $0.05 \%$ Tween-20 (PBST), and blocking with the solution BSA $1 \%$ /PBST, overnight hybridization with human Rad51 protein (NP_002866 Creative Biomart, NY) was performed. Test compounds were added in dose-response from 0.01 to $100 \mu \mathrm{M}$ in triplicate with constant DMSO 1\%. Antibody raised against Rad51 (Millipore) and HRP-secondary antibody staining to develop the $3,3^{\prime}, 5,5^{\prime}$-tetramethyllbenzidine signal (Sigma) quenched with $1 \mathrm{M} \mathrm{HCl}$ was used as the assay readout. Colorimetric measure was read on a Victor5 (PerkinElmer) plate Reader. BRC4 and Rad51 were included in the assay as positive controls. Results were analyzed by using GraphPad Software.

Cell Culture. BxPC-3 and Capan-1 cells were grown in RPMI 1640 containing $10 \%$ FBS, $100 \mathrm{U} / \mathrm{mL}$ penicillin/streptomycin, and $4 \mathrm{mM}$ glutamine. All media and supplements were from Sigma-Aldrich.

Crystal Violet Assay. BxPC-3 and Capan- 1 cells $\left(10^{3}-10^{4} /\right.$ well $)$ were plated in 96-well plates and treated. At the end of treatments, the cell culture medium was removed, and the cells were washed by PBS and fixed in glutaraldehyde $1 \%$ in PBS for 15 min at rt and rinsed with PBS several times. Then, the samples were incubated in $0.01 \%$ Crystal Violet for $30 \mathrm{~min}$ and washed in PBS. To each well was added $100 \mu \mathrm{L}$ of cold ethanol $70 \%$ and the absorbance measured in a microplate reader (Bio-Rad, Hercules, CA, USA) at $570 \mathrm{~nm}$. The results were expressed as a percentage of viable cells on the control.

Interaction Index. The interaction index between Olaparib and $\mathbf{2}$ was assessed by applying the following formula, as reported in ref 25 :
[Surviving cells treated with the combination]

$/[$ (Surviving cells treated with Olaparib)

$\times$ (Surviving cells treated with 2$)$ ]

According to ref 25, a result ranging from 0.8 to 1.2 denotes an additive effect. Synergism is indicated by a result $<0.8$; antagonism by a result $>1.2$.

Western Blot. Cell cultures $\left(1.5 \times 10^{6}\right.$ cells $)$ were exposed to Cisplatin $(12 \mu \mathrm{M})$ for $1 \mathrm{~h}$. Cisplatin was then removed, and 2 was added to the culture medium at the doses used in the combination experiments with Olaparib. After $48 \mathrm{~h}$ of incubation, cells were lysed in $100 \mu \mathrm{L}$ of RIPA buffer containing protease and phosphatase inhibitors (Sigma-Aldrich). The cell homogenates were left $30 \mathrm{~min}$ on ice and then centrifuged $15 \mathrm{~min}$ at $10000 \mathrm{~g}$. A total of $50 \mu \mathrm{g}$ of proteins of the supernatants (measured according to Bradford) were loaded into $12 \%$ polyacrylamide gel for electrophoresis. The separated proteins were blotted on a low fluorescent PVDF membrane (GE Lifescience) using a standard apparatus for wet transfer with an electrical field of $80 \mathrm{~mA}$ for $4 \mathrm{~h}$. The blotted membrane was blocked with 5\% BSA in TBSTween and probed with the primary antibody. The antibodies used were rabbit anti- $\gamma \mathrm{H} 2 \mathrm{AX}$ [phospho S139] (Abcam) and rabbit antiActin (Sigma-Aldrich). Binding was revealed by a Cy5-labeled secondary antibody (anti rabbit-IgG, GE Lifescience; anti mouseIgG, Jackson Immuno-Research). All incubation steps were performed according to the manufacturer's instructions. Fluorescence of the blots was assayed with the Pharos FX scanner (BioRad) at a resolution of $100 \mu \mathrm{m}$, using the Quantity One software (BioRad).

Rad51 Silencing in BxPC-3 Cells. Both stable and transient Rad51 silencing were obtained.

For the transient silencing procedure, the sequences of small interfering RNA (siGENOME SMART pool and siGENOME NonTargeting siRNA Pool \#1) were provided by Dharmacon Research, Lafayette, Colorado, USA. Nontargeting siRNA (SCRAMBLED) and siRNA duplexes for Rad51 (10-100 nM) were transfected into BxPC3 cells using Lipofectamine 2000 (Invitrogen) for $5 \mathrm{~h}$, according to the supplier's instructions.

Stable Rad51 silencing procedure:

Transduction of BXPC3 Cells. Lentiviral sh-RNA based plasmids targeting Rad51 were obtained from Sigma-Aldrich (MISSION shRNA DNA Clone TCR). The plasmids were tested for their ability to knock down Rad51 and to cooperate with Olaparib in synthetic lethality assays. Of the plasmids that were validated using these initial assays, we selected one (pLKO.1 Rad51, TRCN0000018877) for the studies described here. The sequence of the short hairpin RNA (shRNA) targeting the human Rad51 (GenBank accession no. NM_002875) was 5'-CCGGGCTGAAGCTATGTTCGCCATTCTCGAGAATGGCGAACATAGCTTCAGCTTTTT-3'. The control lentiviral pLKO.1 contains a scrambled sequence, not targeting known genes. To generate viral particles, human embryonic kidney 293 cells (ATCC) were cotransfetcted with the lentiviral vector and packaging plasmids (VSVg and PAX8).

BxPC3 cells were exposed to lentivirus-containing supernatant for $16 \mathrm{~h}$ in the presence of Polybrene $(8 \mu \mathrm{g} / \mathrm{mL})$. Pools of stable transfectancts were established by selection using $1 \mu \mathrm{g} / \mathrm{mL}$ Puromycin for $72 \mathrm{~h}$ and mantained in a medium containing $1 \mu \mathrm{g} / \mathrm{mL}$ of Puromycin.

Western Blot. The extent of Rad51 knockdown was measured by Western Blot. Whole cell extracts were obtained by lysis in urea buffer (8 M urea, $25 \mathrm{mM}$ Tris- $\mathrm{HCl}$ at $\mathrm{pH} 6.8,1 \mathrm{mM}$ EDTA). Proteins were quantified (Biorad Bradford assay), and $40 \mu \mathrm{g}$ of total proteins were separated by $12 \%$ SDS-polyacrylamide gel electrophoresis (SDSPAGE), blotted onto a nitrocellulose membrane (Whatman, Schleicher, and Schuell), and probed with antibodies anti-Rad51 (sc8349) and antivinculin (Sigma-Aldrich).

siControl (Scrambled) and siRad51 BxPC-3 cells were maintained in a culture medium with $1 \mu \mathrm{g} / \mathrm{mL}$ of Puromycin added.

RT-PCR. RNA was extracted by PureZOL RNA Isolation Reagent (BioRad, CA, USA). Amplification of specific PCR products was performed using the Verso one-step RT-PCR ReddyMix kit (Thermo 
Fisher Scientific, Waltham, MA, USA). $\beta$-actin was used as a housekeeping gene, and the reaction was run with both Rad51 and $\beta$-actin primers into the same tube. The annealing temperature was set at $58{ }^{\circ} \mathrm{C}$. The primer sequences were Rad51 Forward 5'CTTTGGCCCACAACCCATTTC-3' and Rad51 Reverse 5'ATGGCCTTTCCTTCACCTCCAC- $3^{\prime}$ and $B$-actin Forward $5^{\prime}$ ATCGTGCGTGACATTAAGGAGAAG- $3^{\prime}$ and $\beta$-actin Reverse $5^{\prime}$ AGGAAGGAAGGCTGGAAGAGTG-3'. PCR products were loaded onto a $1 \%$ agarose gel, run into an electrophoresis chamber, stained by ethidium bromide, and visualized with a UV-transilluminator. Bands were analyzed with a Kodak Electrophoresis Detection and Analysis System (EDAS 290, Eastman Kodak Company, Rochester, NY, USA).

Immunofluorescence. Cells were grown on coverslips. Control, treated, and transfected cells were fixed in formalin 1\% in PBS for 20 min and then in ethanol 70\%, air-dried, and washed twice in PBS. The samples were incubated in 10\% Bovine Serum Albumin (BSA) (SigmaAldrich, USA) in PBS for $30 \mathrm{~min}$ at $37{ }^{\circ} \mathrm{C}$ and subsequently in the primary antibody, rabbit polyclonal antibody anti $\gamma \mathrm{H} 2 \mathrm{AX}$ (1:1000 in $1 \%$ BSA in PBS), or anti Rad51 (1:500 in 1\% BSA in PBS), overnight at $4{ }^{\circ} \mathrm{C}$. After washing, the samples were incubated in antirabbitrhodamine $(1: 500,1 \%$ BSA in PBS) or antirabbit-FITC conjugated secondary antibody (1:1000 1\% BSA in PBS) for $1 \mathrm{~h}$ at $37^{\circ} \mathrm{C}$, washed, air-dried, and mounted in a solution 1:500 DAPI in DABCO. The samples were analyzed with a Nikon fluorescent microscope equipped with filters for FITC, TRITC, and DAPI.

Enzymatic Assays. Assays on proteases and phosphatases were run by CEREP (Le Bois l'Eveque, FR), as previously described in references reported in Table 2.

\section{ASSOCIATED CONTENT}

\section{S Supporting Information}

The Supporting Information is available free of charge on the ACS Publications website at DOI: 10.1021/acschembio.7b00707.

Supporting Figures S1-S4, virtual screening protocol, and experimental details of all synthetic procedures together with characterization data for all intermediates and final compounds (PDF)

\section{AUTHOR INFORMATION}

\section{Corresponding Author}

*Phone: +39010 71781530. E-mail: andrea.cavalli@iit.it.

\section{ORCID $\odot$}

Andrea Cavalli: 0000-0002-6370-1176

Notes

The authors declare no competing financial interest.

\section{ACKNOWLEDGMENTS}

We thank the Italian Institute of Technology and Bologna University for financial support. We thank G. Fox for copyediting and proofreading the manuscript. We thank P. G. Pelicci for useful discussions.

\section{DEDICATION}

This paper is dedicated to the memory of Paola (1979-2015).

\section{REFERENCES}

(1) McLornan, D. P., List, A., and Mufti, G. J. (2014) Applying Synthetic Lethality for the Selective Targeting of Cancer. N. Engl. J. Med. 371, 1725-1735.

(2) Dobzhansky, T. (1946) Genetics of Natural Populations. XIII. Recombination and Variability in Populations of Drosophila Pseudoobscura. Genetics 31, 269-290.

(3) Lord, C. J., and Ashworth, A. (2016) BRCAness revisited. Nat. Rev. Cancer 16, 110-120.
(4) Chalasani, P., and Livingston, R. (2013) Differential chemotherapeutic sensitivity for breast tumors with "BRCAness": a review. Oncologist 18, 909-916.

(5) Deeks, E. D. (2015) Olaparib: first global approval. Drugs 75, 231-240.

(6) Kaelin, W. G. (2009) Synthetic lethality: a framework for the development of wiser cancer therapeutics. Genome Med. 1, 99.

(7) Fang, B. (2014) Development of synthetic lethality anticancer therapeutics. J. Med. Chem. 57, 7859-7873.

(8) Dedes, K. J., Wilkerson, P. M., Wetterskog, D., Weigelt, B., Ashworth, A., and Reis-Filho, J. S. (2011) Synthetic lethality of PARP inhibition in cancers lacking BRCA1 and BRCA2 mutations. Cell Cycle $10,1192-1199$

(9) Wyman, C., and Kanaar, R. (2004) Homologous recombination: Down to the wire. Curr. Biol. 14, 629-631.

(10) Roy, R., Chun, J., and Powell, S. N. (2011) BRCA1 and BRCA2: different roles in a common pathway of genome protection. Nat. Rev. Cancer 12, 68-78.

(11) Davies, A. a, Masson, J. Y., Mcllwraith, M. J., Stasiak, A. Z., Stasiak, A., Venkitaraman, A. R., and West, S. C. (2001) Role of BRCA2 in control of the RAD51 recombination and DNA repair protein. Mol. Cell 7, 273-282.

(12) Carreira, A., Hilario, J., Amitani, I., Baskin, R. J., Shivii, M. K. K., Venkitaraman, A. R., and Kowalczykowski, S. C. (2009) The BRC repeats of BRCA2 modulate the DNA-binding selectivity of RAD51. Cell 136, 1032-1043.

(13) Pellegrini, L., Yu, D. S., Lo, T., Anand, S., Lee, M., Blundell, T. L., and Venkitaraman, A. R. (2002) Insights into DNA recombination from the structure of a RAD51-BRCA2 complex. Nature 420, 287293

(14) Rajendra, E., and Venkitaraman, A. R. (2010) Two modules in the BRC repeats of BRCA2 mediate structural and functional interactions with the RAD51 recombinase. Nucleic Acids Res. 38, $82-96$.

(15) Nomme, J., Renodon-Cornière, A., Asanomi, Y., Sakaguchi, K., Stasiak, A. Z., Stasiak, A., Norden, B., Tran, V., and Takahashi, M. (2010) Design of potent inhibitors of human RAD51 recombinase based on BRC motifs of BRCA2 protein: modeling and experimental validation of a chimera peptide. J. Med. Chem. 53, 5782-5791.

(16) Abbott, D. W., Freeman, M. L., and Holt, J. T. (1998) Doublestrand break repair deficiency and radiation sensitivity in BRCA2 mutant cancer cells. J.Natl.Cancer Inst. 90, 978-985.

(17) Jdey, W., Thierry, S., Russo, C., Devun, F., Al Abo, M., NoguiezHellin, P., Sun, J.-S., Barillot, E., Zinovyev, A., Kuperstein, I., Pommier, Y., and Dutreix, M. (2017) Drug-Driven Synthetic Lethality: Bypassing Tumor Cell Genetics with a Combination of AsiDNA and PARP Inhibitors. Clin. Cancer Res. 23, 1001-1011.

(18) Mistrik, M., Oplustilova, L., Lukas, J., and Bartek, J. (2009) Lowdose DNA damage and replication stress responses quantified by optimized automated single-cell image analysis. Cell Cycle 8, 25922599.

(19) Vernole, P., Muzi, A., Volpi, A., Dorio, A. S., Terrinoni, A., Shah, G. M., and Graziani, G. (2009) Inhibition of homologous recombination by treatment with BVDU (brivudin) or by RAD51 silencing increases chromosomal damage induced by bleomycin in mismatch repair-deficient tumour cells. Mutat. Res., Fundam. Mol. Mech. Mutagen. 664, 39-47.

(20) Maxwell, K. N., and Domchek, S. M. (2012) Cancer treatment according to BRCA1 and BRCA2 mutations. Nat. Rev. Clin. Oncol. 9, $520-528$.

(21) Banerjee, S., Kaye, S. B., and Ashworth, A. (2010) Making the best of PARP inhibitors in ovarian cancer. Nat. Rev. Clin. Oncol. 7, $508-519$.

(22) Montoni, A., Robu, M., Pouliot, É., and Shah, G. M. (2013) Resistance to PARP-inhibitors in cancer therapy. Front. Pharmacol. 4 (FEB), 1-7.

(23) Liu, Y., Burness, M. L., Martin-Trevino, R., Guy, J., Bai, S., Harouaka, R., Brooks, M. D., Shang, L., Fox, A., Luther, T. K., Davis, A., Baker, T. L., Colacino, J., Clouthier, S. G., Shao, Z., Wicha, M. S., 
and Liu, S. (2017) RAD51 Mediates Resistance of Cancer Stem Cells to PARP Inhibition in Triple-Negative Breast Cancer. Clin. Cancer Res. 23, 514-522.

(24) Klein, H. L. (2008) The consequences of Rad51 overexpression for normal and tumor cells. DNA Repair 7, 686-693.

(25) Dos Santos Ferreira, A. C., Fernandes, R. A., Kwee, J. K., and Klumb, C. E. (2012) Histone deacetylase inhibitor potentiates chemotherapy-induced apoptosis through Bim upregulation in Burkitt's lymphoma cells. J. Cancer Res. Clin. Oncol. 138, 317-325.

(26) Barrett, A. J., and Kirschke, H. (1981) Cathepsin B, cathepsin H, and cathepsin L. Methods Enzymol. 80, 535-561.

(27) Yasuda, Y., Kageyama, T., Akamine, A., Shibata, M., Kominami, E., Uchiyama, Y., and Yamamoto, K. (1999) Characterization of new fluorogenic substrates for the rapid and sensitive assay of cathepsin $\mathrm{E}$ and cathepsin D. J. Biochem. 125, 1137-1143.

(28) Brömme, D., and Mcgrath, M. E. (1996) High level expression and crystallization of recombinant human cathepsin S. Protein Sci. 5, 789-791.

(29) Nagase, H., Fields, C. G., and Fields, G. B. (1994) Design and characterization of a fluorogenic substrate selectively hydrolyzed by stromelysin 1 (matrix metalloproteinase-3). J. Biol. Chem. 269, 2095220957.

(30) Tierno, M. B., Johnston, P. a, Foster, C., Skoko, J. J., Shinde, S. N., Shun, T. Y., and Lazo, J. S. (2007) Development and optimization of high-throughput in vitro protein phosphatase screening assays. Nat. Protoc. 2, 1134-1144.

(31) Davis, M. I., Ronesi, J., and Lovinger, D. M. (2003) A predominant role for inhibition of the adenylate cyclase/protein kinase A pathway in ERK activation by cannabinoid receptor 1 in N1E-115 neuroblastoma cells. J. Biol. Chem. 278, 48973-48980.

(32) Montalibet, J., Skorey, K. I., and Kennedy, B. P. (2005) Protein tyrosine phosphatase: Enzymatic assays. Methods 35, 2-8.

(33) Welte, S., Baringhaus, K. H., Schmider, W., Müller, G., Petry, S., and Tennagels, N. (2005) 6,8-Difluoro-4-methylumbiliferyl phosphate: A fluorogenic substrate for protein tyrosine phosphatases. Anal. Biochem. 338, 32-38. 